\title{
THE STATIONARY DISTRIBUTIONS OF TWO CLASSES OF REFLECTED ORNSTEIN-UHLENBECK PROCESSES
}

\author{
XIAOYU XING, ${ }^{*}$ Hebei University of Technology \\ WEI ZHANG, ${ }^{* *}$ Central South University \\ YONGJIN WANG, ${ }^{* * *}$ Nankai University
}

\begin{abstract}
In this paper we consider two classes of reflected Ornstein-Uhlenbeck (OU) processes: the reflected OU process with jumps and the Markov-modulated reflected OU process. We prove that their stationary distributions exist. Furthermore, for the jump reflected OU process, the Laplace transform (LT) of the stationary distribution is given. As for the Markov-modulated reflected OU process, we derive an equation satisfied by the LT of the stationary distribution.

Keywords: Ornstein-Uhlenbeck process; reflected process; Markov-modulated process; stationary distribution
\end{abstract}

2000 Mathematics Subject Classification: Primary 91B70

\section{Introduction}

Reflected Ornstein-Uhlenbeck (OU) processes are often used to model some typical statedependent queues. They usually serve as suitable approximations for queueing systems with reneging or for multiserver loss models; cf. [10], [11], and [13]. Explicit properties of the reflected OU process can be found in Ward and Glynn [12], where the authors studied the transient structure, the first hitting time, and the stationary distribution. We note that the OU process with jumps and the Markov-modulated OU process have been applied in modeling queues and financial problems. For the OU process with jumps, Novikov [7] derived the Laplace transform (LT) of the first passage time by using the martingale method. Furthermore, Patie [9] obtained the joint distribution of the first passage time and the primitive stopped at the first passage time. As for the Markov-modulated OU process, the ergodicity conditions and the tail estimate of the stationary distribution can be found in [3] and [5] .

Now we give the expression of the reflected OU process mentioned above. A stochastic process $\left\{X_{t}\right\}_{t \geq 0}$ is called a 'reflected' OU process with jumps if

$$
\mathrm{d} X_{t}=-\lambda X_{t} \mathrm{~d} t+\mathrm{d} Y_{t}+\mathrm{d} L_{t}
$$

with $X_{0}=x_{0} \geq 0$, where $\left\{Y_{t}\right\}_{t \geq 0}$ is a spectrally positive Lévy process and $\left\{L_{t}\right\}_{t \geq 0}$ is the minimal, nondecreasing continuous process which makes $X_{t} \geq 0$ for all $t \geq 0$. Here $\left\{L_{t}\right\}_{t \geq 0}$

Received 6 June 2008; revision received 17 March 2009.

* Postal address: School of Sciences, Hebei University of Technology, Beichen District, Tianjin, 300401, P. R. China. Email address: researchandcommunication@gmail.com Supported by NSF 10671052 .

** Postal address: School of Mathematical Sciences, Central South University, Changsha, 410075, P. R. China.

*** Postal address: School of Business, Nankai University, Tianjin, 300071, P. R. China. 
increases on only $\left\{t: X_{t}=0\right\}$, so

$$
\int_{0}^{\infty} \mathbf{1}_{\left\{X_{t}>0\right\}} \mathrm{d} L_{t}=0 .
$$

The other reflected OU process, denoted by $\left\{\tilde{X}_{t}\right\}_{t \geq 0}$, is the Markov-modulated reflected OU process, which evolves like

$$
\mathrm{d} \tilde{X}_{t}=-\left(b\left(J_{t}\right)+\lambda\left(J_{t}\right) \tilde{X}_{t}\right) \mathrm{d} t+\sigma\left(J_{t}\right) \mathrm{d} B_{t}+\mathrm{d} \tilde{L}_{t} ; \quad\left(\tilde{X}_{0}, J_{0}\right)=(x, i), \quad x \geq 0, i \in \mathbb{E},
$$

where $\left\{J_{t}\right\}_{t \geq 0}$ is a Markov process with finite state space $\mathbb{E}$, and $b(\cdot), \lambda(\cdot)$, and $\sigma(\cdot)$ are positive functions on $\mathbb{E}$. The reflection $\left\{\tilde{L}_{t}\right\}_{t \geq 0}$ is defined analogously to $\left\{L_{t}\right\}_{t \geq 0}$.

The purpose of this paper is to study the stationary distributions of the reflected OU process defined above, since they are important performance measures in queueing theory. The remainder of the paper is organized as follows. In Section 2 we consider the reflected OU process with jumps. We prove that the stationary distribution exists uniquely. By taking advantage of the infinitesimal generator and martingale method, we derive the explicit LT of the stationary distribution. In particular, if the process degenerates to a reflected OU process without jumps, our result is consistent with that given in [12]. Section 3 is devoted to the Markov-modulated reflected OU process. As well as achieving the existence and uniqueness of the stationary distribution, we derive a differential equation satisfied by the LT of the stationary distribution. In the special model with $\lambda=\sigma^{2}, b=0$, and $\mathbb{E}=\{1,2\}$, we obtain an explicit Laplace expression for the stationary distribution.

\section{The reflected OU process with jumps}

We begin by introducing some notation. Suppose that $\left\{B_{t}\right\}_{t \geq 0}$ is a standard Brownian motion, that $N(\mathrm{~d} x, \mathrm{~d} s)$ is a Poisson random measure supported on $[0, \infty) \times[0, \infty)$, and that $b$ and $\sigma$ are two constants. Define $\left\{Y_{t}\right\}_{t \geq 0}$ by

$$
Y_{t}=b t+\sigma B_{t}+\int_{0}^{t} \int_{0}^{1} x \tilde{N}(\mathrm{~d} x, \mathrm{~d} s)+\int_{0}^{t} \int_{1}^{\infty} x N(\mathrm{~d} x, \mathrm{~d} s)
$$

where $\tilde{N}(\mathrm{~d} x, \mathrm{~d} s)=N(\mathrm{~d} x, \mathrm{~d} s)-v(\mathrm{~d} x) \mathrm{d} s$, and $v(\mathrm{~d} x)$ denotes the Lévy measure satisfying $\int_{0}^{\infty}\left(x^{2} \wedge 1\right) v(\mathrm{~d} x)<\infty$. Let $\psi(\cdot)$ be the characteristic exponent of $\left\{Y_{t}\right\}_{t \geq 0}$. Then we have

$$
\begin{aligned}
\psi(\alpha) & =\log \mathrm{E} \exp \left\{-\alpha Y_{1}\right\} \\
& =-b \alpha+\frac{1}{2} \sigma^{2} \alpha^{2}+\int_{0}^{\infty}\left(\mathrm{e}^{-\alpha x}-1+\alpha x \mathbf{1}_{[0,1]}(x)\right) \nu(\mathrm{d} x), \quad \alpha>0 .
\end{aligned}
$$

Here $\left\{X_{t}\right\}_{t \geq 0}$ is a reflected OU process driven by the Lévy process $\left\{Y_{t}\right\}_{t \geq 0}$, that is,

$$
\mathrm{d} X_{t}=-\lambda X_{t} \mathrm{~d} t+\mathrm{d} Y_{t}+\mathrm{d} L_{t}
$$

with $X_{0}=x \geq 0$ and $\lambda>0$, where $\left\{L_{t}\right\}_{t \geq 0}$ is the minimal nondecreasing continuous process which makes $X_{t} \geq 0$ and increases only when $X_{t}$ is 0 .

Theorem 2.1. Assume that $\sigma \neq 0, \int_{1}^{\infty} x v(\mathrm{~d} x)<\infty$, and that $\mathrm{E}\left(Y_{1}^{+}\right)^{\delta}<\infty$ for some $\delta \in$ $(0,1)$. Then $\left\{X_{t}\right\}_{t \geq 0}$ has a unique stationary distribution. 
Proof. In the case $v(0, \infty)=0,\left\{X_{t}\right\}_{t \geq 0}$ degenerates to a reflected OU process without jumps. This result has been proved in [12].

In the rest of the proof we assume that $v(0, \infty)>0$. We claim that $\left\{X_{t}\right\}_{t \geq 0}$ is regenerative and the mean regeneration cycle is finite. Without loss of generality, we assume that there exists a $\varepsilon>0$ such that $\nu(\varepsilon, \infty)>0$. Let $T_{0}^{\varepsilon}=\inf \left\{t>0: X_{t}>\varepsilon \mid X_{0}=0\right\}$ and $T_{\varepsilon}^{0}=\inf \left\{t: t>0, X_{t+T_{0}^{\varepsilon}}=0 \mid X_{0}=0\right\}$. Thus, $T_{0}^{\varepsilon}$ and $T_{\varepsilon}^{0}$ form a typical regeneration cycle, which involves a 'first passage' from 0 to $\varepsilon$, followed by a 'first passage' from the horizon above $\varepsilon$ to 0 .

Observe that all the jumps with sizes larger than $\varepsilon$ constitute a compound Poisson process. The first jump of the compound Poisson process is assumed to occur at $T_{1}$ with size $S$. Since $S>\varepsilon$, so $T_{0}^{\varepsilon} \leq T_{1}$. In addition, since $T_{1}$ is exponentially distributed, it follows that $\mathrm{E} T_{0}^{\varepsilon}<\infty$.

In order to prove that $\mathrm{E} T_{\varepsilon}^{0}<\infty$, we use Theorem 4 of [7], which is established for the jump OU process without reflection. Note that, starting from $T_{0}^{\varepsilon},\left\{X_{t}\right\}_{t \geq 0}$ moves freely, like a jump OU process without reflection, until reaching the zero level, so the theorem is valid in our case. We present it here, slightly adapted our setting. Define $\tau_{x}^{0}=\inf \left\{t \geq 0: X_{t} \leq 0 \mid X_{0}=x\right\}$. If $\sigma \neq 0$ and $\mathrm{E}\left(Y_{1}^{+}\right)^{\delta}<\infty$ for some $\delta \in(0,1)$, then

$$
\mathrm{E} \tau_{x}^{0}=\frac{1}{\lambda} \int_{0}^{\infty}\left(1-\mathrm{e}^{-u x}\right) u^{-1} \mathrm{e}^{-\varphi(u)} \mathrm{d} u<\infty .
$$

This suggests that

$$
\mathrm{E} T_{\varepsilon}^{0}=\frac{1}{\lambda} \mathrm{E} \int_{0}^{\infty}\left(1-\exp \left\{-u X_{T_{0}^{\varepsilon}}\right\}\right) u^{-1} \mathrm{e}^{-\varphi(u)} \mathrm{d} u .
$$

It is not hard to check that $\int_{1}^{\infty} u^{-1} \mathrm{e}^{-\varphi(u)} \mathrm{d} u<\infty$. For our purpose, it suffices to prove that

$$
\mathrm{E} \int_{0}^{1}\left(1-\exp \left\{-u X_{T_{0}^{\varepsilon}}\right\}\right) u^{-1} \mathrm{e}^{-\varphi(u)} \mathrm{d} u<\infty .
$$

By the assumption that $\int_{1}^{\infty} x v(\mathrm{~d} x)<\infty$,

$$
\mathrm{E} \int_{0}^{1}\left(1-\exp \left\{-u X_{T_{0}^{\varepsilon}}\right\}\right) u^{-1} \mathrm{e}^{-\varphi(u)} \mathrm{d} u \leq \mathrm{E} X_{T_{0}^{\varepsilon}} \int_{0}^{1} \mathrm{e}^{-\varphi(u)} \mathrm{d} u<\infty .
$$

Based on the above discussion, $\left\{X_{t}\right\}_{t \geq 0}$ is a regenerative process, and the mean length of a regeneration cycle is equal to $\mathrm{E} T_{0}^{\varepsilon}+\mathrm{E} T_{\varepsilon}^{0}<\infty$. Thus, $\left\{X_{t}\right\}_{t \geq 0}$ has a unique stationary distribution.

Theorem 2.2. Assume that the stationary distribution of $\left\{X_{t}\right\}_{t \geq 0}$ exists and that $g(x)$ is the stationary density. Define $\hat{g}(\alpha):=\int_{0}^{\infty} \mathrm{e}^{-\alpha x} g(x) \mathrm{d} x, \alpha>0$. Then we have

$$
\hat{g}(\alpha)=\frac{\int_{\alpha}^{\infty} \exp \left\{-\int_{\alpha}^{s}(\psi(u) / \lambda u) \mathrm{d} u\right\} \mathrm{d} s}{\int_{0}^{\infty} \exp \left\{-\int_{0}^{s}(\psi(u) / \lambda u) \mathrm{d} u\right\} \mathrm{d} s} .
$$

Proof. Let $S([0 ; \infty), R)$ be the Schwartz space of rapidly decreasing functions. More precisely, $S([0 ; \infty), R)$ is a linear space of all $f(x) \in C^{\infty}([0, \infty) ; R)$ satisfying

$$
\sup _{x \in[0, \infty)}\left|x^{n_{1}} f^{\left(n_{2}\right)}(x)\right|<\infty \quad \text { for all } n_{1}, n_{2} \in \mathbb{N} .
$$


Applying Itô's formula (see [1, Theorem 4.4.7, p. 226]) for Lévy-type stochastic integrals, for any $f(x) \in S([0, \infty), R)$,

$$
\begin{aligned}
\mathrm{d} f\left(X_{t}\right)= & {\left[-\lambda X_{t} f^{\prime}\left(X_{t-}\right)+b f^{\prime}\left(X_{t-}\right)+\frac{1}{2} \sigma^{2} f^{\prime \prime}\left(X_{t-}\right)\right] \mathrm{d} t+f^{\prime}\left(X_{t-}\right) \mathrm{d} L_{t} } \\
& +\int_{0}^{\infty}\left[f\left(X_{t-}+x\right)-f\left(X_{t-}\right)-x f^{\prime}\left(X_{t-}\right) \mathbf{1}_{[0,1]}(x)\right] v(\mathrm{~d} x) \mathrm{d} t \\
& +\int_{0}^{\infty}\left[f\left(X_{t-}+x\right)-f\left(X_{t-}\right)\right] \tilde{N}(\mathrm{~d} x, \mathrm{~d} t)+\sigma f^{\prime}\left(X_{t-}\right) \mathrm{d} B_{t} .
\end{aligned}
$$

Owing to the definition of $S([0, \infty), R)$, the integrals in (2.2) are well defined. It is easy to see that $\left\{X_{t}\right\}_{t \geq 0}$ is a Markov process with infinitesimal generator defined by

$$
\begin{aligned}
\mathcal{A} f(x)= & \lim _{t \rightarrow 0} \frac{\mathrm{E}_{x} f\left(X_{t}\right)-\mathrm{E}_{x} f\left(X_{0}\right)}{t} \\
= & -\lambda x f^{\prime}(x)+b f^{\prime}(x)+\frac{1}{2} \sigma^{2} f^{\prime \prime}(x)+f^{\prime}(0) l_{x} \\
& +\int_{0}^{\infty}\left[f(x+y)-f(x)-y f^{\prime}(x) \mathbf{1}_{[0,1]}(y)\right] v(\mathrm{~d} y),
\end{aligned}
$$

where $l_{x}:=\lim _{t \rightarrow 0} E_{x} L_{t} / t$. To see that $l_{x}$ is well defined for each $x$, we only need to show that $l_{0}$ is well defined, since $l_{x}$ is nonincreasing with respect to $x$. Note that $L_{t}$ is the local time of $\left\{X_{t}\right\}_{t \geq 0}$. By Corollary 6 of [2, p. 112], there is some constant $c$ such that $\int_{0}^{t} \mathbf{1}_{\left\{X_{s}=0\right\}} \mathrm{d} s=c L_{t}$, so $l_{0}$ is a finite constant. By the martingale representation theorem we obtain

$$
\mathrm{E}_{x} f\left(X_{t}\right)-f(x)=\int_{0}^{t} \mathrm{E}_{x}(\mathcal{A} f)\left(X_{s}\right) \mathrm{d} s .
$$

This suggests that if $g(x)$ is the stationary density function then

$$
\begin{aligned}
0 & =\int_{0}^{\infty} g(x)\left(\mathrm{E}_{x} f\left(X_{t}\right)\right) \mathrm{d} x-\int_{0}^{\infty} g(x)(f(x)) \mathrm{d} x \\
& =\int_{0}^{\infty} g(x)\left[\int_{0}^{t} \mathrm{E}_{x}(\mathcal{A} f)\left(X_{s}\right) \mathrm{d} s\right] \mathrm{d} x \\
& =\int_{0}^{t} \int_{0}^{\infty}(\mathcal{A} f)(x) g(x) \mathrm{d} x \mathrm{~d} s .
\end{aligned}
$$

So

$$
\int_{0}^{\infty}(\mathcal{A} f)(x) g(x) \mathrm{d} x=0 \text { for all } f \in S([0, \infty), R) .
$$

Specifically, we select $f(x)=-\mathrm{e}^{-\alpha x} / \alpha$. By a straightforward calculation we establish that

$$
\begin{aligned}
\int_{0}^{\infty}(\mathcal{A} f)(x) g(x) \mathrm{d} x \\
=-\lambda \int_{0}^{\infty} x \mathrm{e}^{-\alpha x} g(x) \mathrm{d} x+b \int_{0}^{\infty} \mathrm{e}^{-\alpha x} g(x) \mathrm{d} x-\frac{1}{2} \sigma^{2} \alpha \int_{0}^{\infty} \mathrm{e}^{-\alpha x} g(x) \mathrm{d} x \\
\quad+\int_{0}^{\infty} l_{x} g(x) \mathrm{d} x-\frac{1}{\alpha} \int_{0}^{\infty} \mathrm{e}^{-\alpha x} g(x) \mathrm{d} x \int_{0}^{\infty}\left[\mathrm{e}^{-\alpha y}-1+\alpha y \mathbf{1}_{[0,1]}(y)\right] \nu(\mathrm{d} y) \\
=\lambda \hat{g}^{\prime}(\alpha)+\hat{g}(\alpha)\left[b-\frac{1}{2} \alpha \sigma^{2}-\frac{1}{\alpha} \int_{0}^{\infty}\left(\mathrm{e}^{-\alpha y}-1+\alpha y \mathbf{1}_{[0,1]}(y)\right) \nu(\mathrm{d} y)\right]+p \\
=\lambda \hat{g}^{\prime}(\alpha)-\frac{\psi(\alpha)}{\alpha} \hat{g}(\alpha)+p,
\end{aligned}
$$


where $p:=\int_{0}^{\infty} l_{x} g(x) \mathrm{d} x$. Substituting this identity into (2.3) yields

$$
\hat{g}^{\prime}(\alpha)-\frac{\psi(\alpha)}{\lambda \alpha} \hat{g}(\alpha)+\frac{p}{\lambda}=0, \quad \alpha \in(0, \infty)
$$

Note that $\hat{g}(0)=1$. The unique solution of (2.4) is given by

$$
\hat{g}(\alpha)=C(\alpha) \exp \left\{\int_{0}^{\alpha} \frac{\psi(s)}{\lambda s} \mathrm{~d} s\right\},
$$

where

$$
C(\alpha):=1-\frac{p}{\lambda} \int_{0}^{\alpha} \exp \left\{-\int_{0}^{s} \frac{\psi(u)}{\lambda u} \mathrm{~d} u\right\} \mathrm{d} s .
$$

Finally, we end the proof by identifying the constant $p$. Owing to the fact that $\hat{g}(\alpha) \rightarrow 0$, $\exp \left\{\int_{0}^{\alpha}(\psi(s) / \lambda s) \mathrm{d} s\right\} \rightarrow \infty$ as $\alpha \rightarrow \infty$. We deduce that $C(\alpha) \rightarrow 0$ as $\alpha \rightarrow \infty$, which implies that

$$
\frac{p}{\lambda} \int_{0}^{\infty} \exp \left\{-\int_{0}^{s} \frac{\psi(u)}{\lambda u} \mathrm{~d} u\right\} \mathrm{d} s=1 .
$$

Hence, $p=\lambda\left[\int_{0}^{\infty} \exp \left\{-\int_{0}^{s}(\psi(u) / \lambda u) \mathrm{d} u\right\} \mathrm{d} s\right]^{-1}$. Returning to (2.5), by substituting the value of $p$ into $C(\alpha)$ we have

$$
\hat{g}(\alpha)=\frac{\int_{\alpha}^{\infty} \exp \left\{-\int_{\alpha}^{s}(\psi(u) / \lambda u) \mathrm{d} u\right\} \mathrm{d} s}{\int_{0}^{\infty} \exp \left\{-\int_{0}^{s}(\psi(u) / \lambda u) \mathrm{d} u\right\} \mathrm{d} s} .
$$

This completes the proof.

Example 2.1. Let $Y_{t}=b t+\sigma B_{t}$. Then $X_{t}$ becomes an ordinary reflected OU process driven only by a Brownian motion:

$$
\mathrm{d} X_{t}=\left(b-\lambda X_{t}\right) \mathrm{d} t+\sigma \mathrm{d} B_{t}+\mathrm{d} L_{t} .
$$

According to Theorem 2.2, the Laplace transform of the stationary density is given by

$$
\hat{g}(\alpha)=\frac{\int_{\alpha}^{\infty} \exp \left\{\left(4 b(s-\alpha)-\sigma^{2}\left(s^{2}-\alpha^{2}\right)\right) / 4 \lambda\right\} \mathrm{d} s}{\int_{0}^{\infty} \exp \left\{\left(4 b s-\sigma^{2} s^{2}\right) / 4 \lambda\right\} \mathrm{d} s} .
$$

On the other hand, Ward and Glynn [12] proved that

$$
g(x)=\sqrt{\frac{2 \lambda}{\sigma^{2}}} \frac{\phi\left(\sqrt{\left(2 \lambda / \sigma^{2}\right)(x-b / \lambda)^{2}}\right)}{1-\Phi\left(-\sqrt{2 b^{2} / \lambda \sigma^{2}}\right)},
$$

where $\phi$ and $\Phi$ are the density and distribution functions of $N(0,1)$ random variables. By performing LTs of both sides of (2.7), we wish to prove that our result (2.6) agrees with (2.7). In fact, the LT of $\exp \left\{-a t^{2}\right\}, a>0$, is

$$
\frac{1}{2} \sqrt{\frac{\pi}{a}} \exp \left\{\frac{\alpha^{2}}{4 a}\right\} \operatorname{Erfc}\left(\frac{\alpha}{2 \sqrt{a}}\right)
$$

where

$$
\operatorname{Erfc}(x)=\frac{2}{\sqrt{\pi}} \int_{x}^{\infty} \exp \left\{-t^{2}\right\} \mathrm{d} t
$$


see $\left[8\right.$, pp. 43, 418] for the transform and the error integral $\operatorname{Erfc}(x)$. Let $a_{1}=\lambda / \sigma^{2}$ and $a_{2}=b / \lambda$. Then

$$
\begin{aligned}
& \int_{0}^{\infty} \mathrm{e}^{-\alpha x} \phi\left(\sqrt{\frac{2 \lambda}{\sigma^{2}}\left(x-\frac{b}{\lambda}\right)^{2}}\right) \mathrm{d} x \\
& =\frac{1}{\sqrt{2 \pi}} \int_{0}^{\infty} \mathrm{e}^{-\alpha x} \exp \left\{-a_{1}\left(x-a_{2}\right)^{2}\right\} \mathrm{d} x \\
& =\frac{1}{\sqrt{2 \pi}} \exp \left\{-a_{1} a_{2}^{2}\right\} \int_{0}^{\infty} \exp \left\{-\left(\alpha-2 a_{1} a_{2}\right) x\right\} \exp \left\{-a_{1} x^{2}\right\} \mathrm{d} x \\
& =\frac{1}{2 \sqrt{2 a_{1}}} \exp \left\{-a_{1} a_{2}^{2}\right\} \exp \left\{\frac{\left(\alpha-2 a_{1} a_{2}\right)^{2}}{4 a_{1}}\right\} \operatorname{Erfc}\left(\frac{\left(\alpha-2 a_{1} a_{2}\right)}{2 \sqrt{a_{1}}}\right) \\
& =\frac{1}{\sqrt{2 \pi a_{1}}} \exp \left\{-a_{1} a_{2}^{2}\right\} \exp \left\{\frac{\left(\alpha-2 a_{1} a_{2}\right)^{2}}{4 a_{1}}\right\} \int_{\left(\alpha-2 a_{1} a_{2}\right) / 2 \sqrt{a_{1}}}^{\infty} \exp \left\{-t^{2}\right\} \mathrm{d} t \\
& =\frac{1}{2 a_{1} \sqrt{2 \pi}} \exp \left\{-a_{1} a_{2}^{2}\right\} \exp \left\{\frac{\left(\alpha-2 a_{1} a_{2}\right)^{2}}{4 a_{1}}\right\} \int_{\alpha}^{\infty} \exp \left\{-\frac{\left(t-2 a_{1} a_{2}\right)^{2}}{4 a_{1}}\right\} \mathrm{d} t \\
& =\frac{1}{2 a_{1} \sqrt{2 \pi}} \exp \left\{-a_{1} a_{2}^{2}\right\} \int_{\alpha}^{\infty} \exp \left\{\frac{4 b(s-\alpha)-\sigma^{2}\left(s^{2}-\alpha^{2}\right)}{4 \lambda}\right\} \mathrm{d} s \text {. }
\end{aligned}
$$

Let $\alpha=0$. The following relation holds:

$$
\begin{aligned}
& \frac{1}{2 a_{1} \sqrt{2 \pi}} \exp \left\{-a_{1} a_{2}^{2}\right\} \int_{0}^{\infty} \exp \left\{\frac{4 b(s-\alpha)-\sigma^{2}\left(s^{2}-\alpha^{2}\right)}{4 \lambda}\right\} \mathrm{d} s \\
& \quad=\int_{0}^{\infty} \phi\left(\sqrt{\frac{2 \lambda}{\sigma^{2}}\left(x-\frac{b}{\lambda}\right)^{2}}\right) \mathrm{d} x \\
& \quad=\frac{1}{\sqrt{2 \pi}} \int_{0}^{\infty} \exp \left\{-\frac{(2 \lambda / \sigma)(x-b / \lambda)^{2}}{2}\right\} \mathrm{d} x \\
& \quad=\frac{1}{\sqrt{2 \pi}} \frac{\sigma}{\sqrt{2 \lambda}} \int_{-\sqrt{2 b^{2} / \lambda \sigma^{2}}}^{\infty} \exp \left\{-\frac{t^{2}}{2}\right\} \mathrm{d} t \\
& =\frac{\sigma}{\sqrt{2 \lambda}}\left[1-\Phi\left(-\sqrt{\frac{2 b^{2}}{\lambda \sigma^{2}}}\right)\right] .
\end{aligned}
$$

Hence, by performing LTs of both sides of (2.7) we obtain (2.6).

Example 2.2. Consider a reflected OU process driven by a standard Brownian motion $\left\{B_{t}\right\}_{t \geq 0}$ and a compound Poisson process $\left\{Z_{t}\right\}_{t \geq 0}$. Specifically, we take the Lévy measure $v(\mathrm{~d} x)=$ $\mathrm{e}^{-r x} \mathrm{~d} x, r>0$. Then

$$
\mathrm{d} X_{t}=-X_{t} \mathrm{~d} t+\mathrm{d} B_{t}+\mathrm{d} Z_{t}+\mathrm{d} L_{t} .
$$

It is easy to see that the Lévy exponent is given by $\psi(\alpha)=\frac{1}{2} \alpha^{2}+r /(\alpha+r)-1$. Thanks to Theorem 2.2, the LT of the stationary density is

$$
\hat{g}(\alpha)=\int_{\alpha}^{\infty} \exp \left\{\frac{\alpha^{2}-s^{2}}{4}\right\} \frac{s+r}{\alpha+r} \mathrm{~d} s / \int_{0}^{\infty} \exp \left\{-\frac{s^{2}}{4}\right\} \frac{s+r}{r} \mathrm{~d} s .
$$


Note that

$$
\begin{aligned}
\int_{\alpha}^{\infty} \exp \left\{\frac{\alpha^{2}-s^{2}}{4}\right\} \frac{s+r}{\alpha+r} \mathrm{~d} s & =2 \int_{0}^{\infty} \exp \left\{-\left(s^{2}+\alpha s\right)\right\} \frac{2 s+\alpha+r}{\alpha+r} \mathrm{~d} s \\
& =\frac{4}{\alpha+r} \int_{0}^{\infty} \mathrm{e}^{-\alpha s} \exp \left\{-s^{2}\right\} s \mathrm{~d} s+2 \int_{0}^{\infty} \mathrm{e}^{-\alpha s} \exp \left\{-s^{2}\right\} \mathrm{d} s
\end{aligned}
$$

Performing inverse LTs of both sides of (2.8), we obtain the density function

$$
g(x)=\left(4 \int_{0}^{x} \exp \left\{-r(x-y)-y^{2}\right\} y \mathrm{~d} y+2 \exp \left\{-s^{2}\right\}\right) / \int_{0}^{\infty} \exp \left\{-\frac{s^{2}}{4}\right\} \frac{s+r}{r} \mathrm{~d} s .
$$

Example 2.2 gives the explicit stationary density for a reflected OU process with Poisson jumps. Generally speaking, for model (2.1), the explicit expression of $g(x)$ is rather difficult to obtain. But with the aid of Theorem 2.2, the Laplace transform of $g(x)$ can be rigorously expressed.

\section{The Markov-modulated reflected OU process}

In this section we consider the stationary distribution of the Markov-modulated reflected OU process. Suppose that $\{J(t)\}_{t \geq 0}$ is an irreducible and recurrent continuous-time Markov chain with finite state space $\mathbb{E}=\{1,2, \ldots, n\}$. Then $\{J(t)\}_{t \geq 0}$ has a unique stationary probability distribution, $\pi=\left(\pi_{1}, \pi_{2}, \ldots, \pi_{n}\right)$, say. The intensity matrix is denoted by $\boldsymbol{Q}=\left(q_{i j}\right)_{i, j \in \mathbb{E}}$.

In this section, $\left\{X_{t}\right\}_{t \geq 0}$ denotes the reflected OU process modulated by $\left\{J_{t}\right\}_{t \geq 0}$. Then the $\left\{X_{t}\right\}_{t \geq 0}$ are formulated using

$$
\mathrm{d} X_{t}=-\left(b\left(J_{t}\right)+\lambda\left(J_{t}\right) X_{t}\right) \mathrm{d} t+\sigma\left(J_{t}\right) \mathrm{d} B_{t}+\mathrm{d} L_{t},
$$

where $\sigma(\cdot), \lambda(\cdot)$, and $b(\cdot)$ are strictly positive functions on $\mathbb{E}$, and $\left\{L_{t}\right\}_{t \geq 0}$ is the minimal nondecreasing process that makes $X_{t} \geq 0$ and increases only when $X_{t}$ is 0 . When $J(\cdot)=j$, $X_{t}$ behaves like a reflected OU process:

$$
\mathrm{d} X_{t}=-\left(b_{j}+\lambda_{j} X_{t}\right) \mathrm{d} t+\sigma_{j} \mathrm{~d} B_{t}+\mathrm{d} L_{t} .
$$

Assume that $X_{0}=0$ and that $\left\{J_{t}\right\}_{t \geq 0}$ starts from the distribution $\pi$. From Itô's formula and (3.1), $\left\{\exp \left\{\int_{0}^{t} \lambda\left(J_{S}\right) \mathrm{d} s\right\} X_{t}\right\}_{t \geq 0}$ has the following representation:

$$
\begin{aligned}
\exp \left\{\int_{0}^{t} \lambda\left(J_{s}\right) \mathrm{d} s\right\} X_{t}= & \int_{0}^{t} \exp \left\{\int_{0}^{s} \lambda\left(J_{u}\right) \mathrm{d} u\right\}\left[-b\left(J_{s}\right) \mathrm{d} s+\sigma\left(J_{s}\right) \mathrm{d} B_{s}\right] \\
& +\int_{0}^{t} \exp \left\{\int_{0}^{s} \lambda\left(J_{u}\right) \mathrm{d} u\right\} \mathrm{d} L_{s} .
\end{aligned}
$$

Let $Y_{t}:=\int_{0}^{t} \exp \left\{\int_{0}^{s} \lambda\left(J_{u}\right) \mathrm{d} u\right\}\left[-b\left(J_{s}\right) \mathrm{d} s+\sigma\left(J_{s}\right) \mathrm{d} B_{s}\right]$ and $R_{t}:=\int_{0}^{t} \exp \left\{\int_{0}^{s} \lambda\left(J_{u}\right) \mathrm{d} u\right\} \mathrm{d} L_{s}$. Then

$$
X_{t}=\exp \left\{-\int_{0}^{t} \lambda\left(J_{s}\right) \mathrm{d} s\right\}\left(Y_{t}+R_{t}\right)
$$

By (3.2) and the definition of $L_{t}, R_{t}$ is a nondecreasing continuous process that makes $Y_{t}+R_{t} \geq 0$. Moreover, $R_{t}$ increases only on the set $\left\{Y_{t}+R_{t}=0\right\}$. According to the solution to the Skorokhod problem (see, e.g. Section 2.2 of [6]), $R_{t}$ is the reflection of $Y_{t}$. Hence, we have the following lemma. 
Lemma 3.1. For the model specified by (3.1), $R_{t}=\int_{0}^{t} \exp \left\{\int_{0}^{s} \lambda\left(J_{u}\right) \mathrm{d} u\right\} \mathrm{d} L_{s}, t \geq 0$, is in fact the reflection of $\left\{Y_{t}\right\}_{t \geq 0}$. That is,

$$
R_{t}=-\inf _{0 \leq s \leq t} Y_{s}, \quad t \geq 0
$$

Hence, $X_{t}$ can be expressed as

$$
X_{t}=\exp \left\{-\int_{0}^{t} \lambda\left(J_{s}\right) \mathrm{d} s\right\}\left[Y_{t}-\inf _{0 \leq s \leq t} Y_{s}\right], \quad t \geq 0 .
$$

Next we prove that $X_{t}$ converges in distribution as $t \rightarrow \infty$, which further implies that the stationary distribution of $\left\{X_{t}\right\}_{t \geq 0}$ exists.

Theorem 3.1. Let $\left\{J_{t}^{*}\right\}_{t \geq 0}$ be the reversed Markov chain of $\left\{J_{t}\right\}_{t \geq 0}$. The initial distribution is $\pi$. Then in the model specified by (3.1), $\left(J_{t}, X_{t}\right)$ converges to

$$
\left(J_{0}^{*}, \max _{t<\infty}\left\{\int_{0}^{t} \exp \left\{-\int_{0}^{s} \lambda\left(J_{u}^{*}\right) \mathrm{d} u\right\}\left[-b\left(J_{s}^{*}\right) \mathrm{d} s+\sigma\left(J_{s}^{*}\right) \mathrm{d} B_{s}\right]\right\}\right)
$$

in distribution. Moreover, the limit is finite almost everywhere. So the stationary distribution exists.

Proof. By Lemma 3.1 we have

$$
\begin{aligned}
X_{T} & =\exp \left\{-\int_{0}^{T} \lambda\left(J_{t}\right) \mathrm{d} t\right\}\left(Y_{T}+R_{T}\right) \\
& =\exp \left\{-\int_{0}^{T} \lambda\left(J_{t}\right) \mathrm{d} t\right\} \max _{0 \leq t \leq T}\left\{Y_{T}-Y_{t}\right\} \\
& =\exp \left\{-\int_{0}^{T} \lambda\left(J_{t}\right) \mathrm{d} t\right\} \max _{0 \leq t \leq T}\left\{\int_{t}^{T} \exp \left\{\int_{0}^{s} \lambda\left(J_{u}\right) \mathrm{d} u\right\}\left[-b\left(J_{s}\right) d s+\sigma\left(J_{s}\right) \mathrm{d} B_{S}\right]\right\} \\
& =\max _{0 \leq t \leq T}\left\{\int_{t}^{T} \exp \left\{-\int_{s}^{T} \lambda\left(J_{u}\right) \mathrm{d} u\right\}\left[-b\left(J_{s}\right) \mathrm{d} s+\sigma\left(J_{s}\right) \mathrm{d} B_{s}\right]\right\} .
\end{aligned}
$$

Recall that $\left\{J_{t}^{*}\right\}_{t \geq 0}$ is the reversed Markov chain of $\left\{J_{t}\right\}_{t \geq 0}$ with initial distribution $\pi$. From (3.3) we have

$$
\left(J_{T}, X_{T}\right) \stackrel{\mathrm{D}}{=}\left(J_{0}^{*}, \max _{0 \leq t \leq T}\left\{\int_{0}^{t} \exp \left\{-\int_{0}^{s} \lambda\left(J_{u}^{*}\right) \mathrm{d} u\right\}\left[-b\left(J_{s}^{*}\right) \mathrm{d} s+\sigma\left(J_{s}^{*}\right) \mathrm{d} B_{s}\right]\right\}\right),
$$

where $\stackrel{\text { D }}{=}$ denotes equality in distribution. Moreover,

$$
\begin{aligned}
X_{T} & \stackrel{\mathrm{D}}{=} \max _{0 \leq t \leq T}\left\{\int_{0}^{t} \exp \left\{-\int_{0}^{s} \lambda\left(J_{u}^{*}\right) \mathrm{d} u\right\}\left[-b\left(J_{s}^{*}\right) \mathrm{d} s+\sigma\left(J_{s}^{*}\right) \mathrm{d} B_{s}\right]\right\} \\
& \leq \max _{0 \leq t \leq T}\left\{\int_{0}^{t} \exp \left\{-\int_{0}^{s} \lambda\left(J_{u}^{*}\right) \mathrm{d} u\right\} \sigma\left(J_{s}^{*}\right) \mathrm{d} B_{s}\right\} \\
& \stackrel{\mathrm{D}}{=} \max _{0 \leq t \leq T}\left\{B_{\int_{0}^{t}} \exp \left\{-2 \int_{0}^{s} \lambda\left(J_{u}^{*}\right) \mathrm{d} u\right\} \sigma^{2}\left(J_{s}^{*}\right) \mathrm{d} s\right. \\
& \leq \max _{0 \leq t \leq \infty}\left\{B_{\int_{0}^{t}} \exp \left\{-2 \int_{0}^{s} \lambda\left(J_{u}^{*}\right) \mathrm{d} u\right\} \sigma^{2}\left(J_{s}^{*}\right) \mathrm{d} s\right. \\
& \leq \max _{0 \leq t \leq \bar{\sigma}^{2} / 2 \underline{\lambda}} B_{t} \\
& <\infty
\end{aligned}
$$


where $\bar{\sigma}=\max _{i \in \mathbb{E}}\{\sigma(i)\}$ and $\underline{\lambda}=\min _{i \in \mathbb{E}}\{\lambda(i)\}$. By (3.4) and (3.5), $X_{t}$ converges to the finite variable

$$
\max _{t<\infty}\left\{\int_{0}^{t} \exp \left\{-\int_{0}^{s} \lambda\left(J_{u}^{*}\right) \mathrm{d} u\right\}\left[-b\left(J_{s}^{*}\right) \mathrm{d} s+\sigma\left(J_{s}^{*}\right) \mathrm{d} B_{s}\right]\right\}
$$

in distribution. This completes the proof.

By $\left(J_{\infty}, X_{\infty}\right)$ we denote the limit of $\left(X_{t}, J_{t}\right)$ in the distribution sense. Define $G_{i}(x)=$ $\mathrm{P}\left(X_{\infty} \leq x, J_{\infty}=i\right)$ for $x \geq 0$ and $i \in \mathbb{E}$. Let $g_{i}(x)$ denote the stationary density at $(x, i)$. Let $\hat{g}_{i}(\alpha)=\int_{0}^{\infty} \mathrm{e}^{-\alpha x} g_{i}(x) \mathrm{d} x$ and $\hat{\boldsymbol{g}}(\alpha)=\left(\hat{g}_{1}(\alpha), \hat{g}_{2}(\alpha), \ldots, \hat{g}_{n}(\alpha)\right)^{\top}$. Then we have the following theorem.

Theorem 3.2. Assume that the stationary distribution of $\left(X_{t}, J_{t}\right)$ exists. Then $\hat{\boldsymbol{g}}(\alpha)$ satisfies the following differential equation:

$$
\boldsymbol{A}(\alpha) \hat{\boldsymbol{g}}(\alpha)-\boldsymbol{B}(\alpha) \hat{\boldsymbol{g}}^{\prime}(\alpha)=\alpha \boldsymbol{P}
$$

where $\boldsymbol{A}(\alpha)=\boldsymbol{Q}^{\top}+\operatorname{diag}\left(\sigma_{i}^{2} \alpha^{2} / 2+b_{i} \alpha\right), \boldsymbol{B}(\alpha)=\operatorname{diag}\left(\lambda_{i} \alpha\right)$, and $\boldsymbol{P}$ is a constant column vector that will be given in the proof below.

Proof. The proof follows by arguments similar to the proof of Theorem 2.2, so we begin with the infinitesimal generator $\mathcal{L}$ of $\left(X_{t}, J_{t}\right)$. Select $f(x, i)$ bounded and twice continuously differentiable in $x$. By Itô's formula,

$$
\begin{aligned}
\mathcal{L} f(x, i)= & \lim _{t \rightarrow 0} \frac{\mathrm{E}_{(x, i)} f\left(X_{t}, J_{t}\right)-\mathrm{E}_{(x, i)} f\left(X_{0}, J_{0}\right)}{t} \\
= & \lim _{t \rightarrow 0} \frac{\left(\mathrm{E}_{(x, i)} f\left(X_{t}, J_{t}\right)-\mathrm{E}_{(x, i)} f\left(X_{t}, J_{0}\right)\right)+\left(\mathrm{E}_{(x, i)} f\left(X_{t}, J_{0}\right)-\mathrm{E}_{(x, i)} f\left(X_{0}, J_{0}\right)\right)}{t} \\
= & \lim _{t \rightarrow 0} t^{-1}\left[\left(1+q_{i i} t\right) \mathrm{E}_{(x, i)} f\left(X_{t}, i\right)+\sum_{j \neq i} q_{i j} t \mathrm{E}_{(x, i)} f\left(X_{t}, j\right)-\mathrm{E}_{(x, i)} f\left(X_{t}, i\right)\right. \\
& \quad-\mathrm{E}_{(x, i)} \int_{0}^{t}\left(b\left(J_{s}\right)+\lambda\left(J_{s}\right) X_{s}\right) f^{\prime}\left(X_{s}, J_{0}\right) \mathrm{d} s+f^{\prime}\left(0, J_{0}\right) \mathrm{E}_{(x, i)} L_{t} \\
& \left.\quad+\mathrm{E}_{(x, i)} \int_{0}^{t} \sigma\left(J_{s}\right) f^{\prime}\left(X_{s}, J_{0}\right) \mathrm{d} B_{s}+\frac{1}{2} \mathrm{E}_{(x, i)} \int_{0}^{t} \sigma^{2}\left(J_{s}\right) f^{\prime \prime}\left(X_{s}, J_{0}\right) \mathrm{d} s\right] \\
= & \sum_{j \in \mathbb{E}} q_{i j} f(x, j)-b_{i} f^{\prime}(x, i)-\lambda_{i} x f^{\prime}(x, i)+\frac{1}{2} \sigma_{i}^{2} f^{\prime \prime}(x, i)+f^{\prime}(0, i) l_{x}^{i}, \quad(3.7)
\end{aligned}
$$

where $l_{x}^{i}:=\lim _{t \rightarrow 0} \mathrm{E}_{(x, i)} L_{t} / t$, and $f^{\prime}(x, i)$ and $f^{\prime \prime}(x, i)$ are the first- and second-order partial derivatives at $x$. The stationary density, $g_{i}(x), i=1,2, \ldots, n$, solves the following equation for all $f(x, i)$ defined above:

$$
\sum_{i=1}^{n} \int_{0}^{\infty}(\mathcal{L} f)(x, i) g_{i}(x) \mathrm{d} x=0 .
$$

We set $f(x, i)=\left(\mathrm{e}^{-\alpha x}-1\right) h(i)$, where $h(i)$ is a bounded function on $\mathbb{E}$. It follows from (3.7) and (3.8) that

$$
\sum_{i=1}^{n} h(i) \int_{0}^{\infty} K_{i}(x) \mathrm{d} x=0
$$


where

$$
\begin{aligned}
K_{i}(x)= & \sum_{j=1}^{n} q_{j i}(\exp \{-\alpha x\}-1) g_{j}(x)+b_{i} \alpha \mathrm{e}^{-\alpha x} g_{i}(x)+\lambda_{i} \alpha x \mathrm{e}^{-\alpha x} g_{i}(x) \\
& +\frac{1}{2} \sigma_{i}^{2} \alpha^{2} \mathrm{e}^{-\alpha x} g_{i}(x)-\alpha l_{x}^{i} g_{i}(x) .
\end{aligned}
$$

Define $p_{i}:=\int_{0}^{\infty} l_{x}^{i} g_{i}(x) \mathrm{d} x$. Note that $\hat{g}_{i}(0)=\pi_{i}$ and $\sum_{j=1}^{n} q_{j i} \pi_{j}=0, i \in \mathbb{E}$. Then the identity above immediately yields

$$
\sum_{i=1}^{n} h(i)\left[\sum_{i=1}^{n} q_{j i} \hat{g}_{j}(\alpha)+b_{i} \alpha \hat{g}_{i}(\alpha)-\lambda_{i} \alpha \hat{g}_{i}^{\prime}(\alpha)+\frac{1}{2} \sigma_{i}^{2} \alpha^{2} \hat{g}_{i}(\alpha)-\alpha p_{i}\right]=0 .
$$

Finally, let $\boldsymbol{P}:=\left(p_{1}, p_{2}, \ldots, p_{n}\right)^{\top}$. Since $h(i)$ is arbitrary, we have

$$
\boldsymbol{A}(\alpha) \hat{\boldsymbol{g}}(\alpha)-\boldsymbol{B}(\alpha) \hat{\boldsymbol{g}}^{\prime}(\alpha)=\alpha \boldsymbol{P} .
$$

Corollary 3.1. Assume that $\boldsymbol{M}_{k}=\left(m_{k}^{1}, m_{k}^{2}, \ldots, m_{k}^{n}\right)^{\top}$ exists for $m_{k}^{i}=\int_{0}^{\infty} x^{k} g_{i}(x) \mathrm{d} x, i \in \mathbb{E}$ and $k \in \mathbb{N}$. Then we have

$$
\begin{gathered}
\boldsymbol{M}_{0}=\left(\pi_{1}, \pi_{2}, \ldots, \pi_{n}\right)^{\top}, \\
\boldsymbol{M}_{1}=\left[\boldsymbol{Q}^{\top}-\operatorname{diag}\left(\lambda_{i}\right)\right]^{-1}\left(\boldsymbol{P}-\operatorname{diag}\left(b_{i}\right) \boldsymbol{\pi}^{\top}\right),
\end{gathered}
$$

and the following recursive relation:

$$
\begin{aligned}
\boldsymbol{M}_{n}= & -\frac{n(n-1)}{2}\left[\boldsymbol{Q}^{\top}-\operatorname{diag}\left(n \lambda_{i}\right)\right]^{-1} \operatorname{diag}\left(\sigma_{i}^{2}\right) \boldsymbol{M}_{n-2} \\
& +n\left[\boldsymbol{Q}^{\top}-\operatorname{diag}\left(n \lambda_{i}\right)\right]^{-1} \operatorname{diag}\left(b_{i}\right) \boldsymbol{M}_{n-1}, \quad n \geq 2 .
\end{aligned}
$$

Proof. Equation (3.9) is trivial. To see (3.11), differentiate (3.6) $k$ times, $k \geq 2$, to obtain

$$
\sum_{l=0}^{k} C_{k}^{l} \boldsymbol{A}^{(l)}(\alpha) \hat{\boldsymbol{g}}^{(k-l)}(\alpha)-\sum_{l=0}^{k} C_{k}^{l} \boldsymbol{B}^{(l)}(\alpha) \hat{\boldsymbol{g}}^{(k-l+1)}(\alpha)=0 .
$$

Letting $\alpha \rightarrow 0$,

$$
\left[\boldsymbol{Q}^{\top}-C_{n}^{1} \operatorname{diag}\left(\lambda_{i}\right)\right] \hat{\boldsymbol{g}}^{(k)}(0)+C_{n}^{1} \operatorname{diag}\left(b_{i}\right) \hat{\boldsymbol{g}}^{(k-1)}(0)+C_{n}^{2} \operatorname{diag}\left(\sigma_{i}^{2}\right) \hat{\boldsymbol{g}}^{(k-2)}(0)=0 .
$$

By the Gershgorin circle theorem (see [4, p. 200]) we can prove the invertibility of $\boldsymbol{Q}^{\top}-$ $C_{n}^{1} \operatorname{diag}\left(\lambda_{i}\right)$. In fact, the Gershgorin circle theorem identifies a region in the complex plane that contains all the eigenvalues of a complex square matrix. For an $n \times n$ matrix $\boldsymbol{A}$, define $R_{i}=\sum_{j=1, j \neq i}^{n}\left|a_{i j}\right|$. Then each eigenvalue of $\boldsymbol{A}$ is in at least one of the disks $\left\{z:\left|z-a_{i i}\right| \leq\right.$ $\left.R_{i}\right\}, i=1,2, \ldots, n$. In particular, if $\left|a_{i i}\right|>R_{i}, i=1,2, \ldots, n$, then each eigenvalue is apart from 0 . So the matrix $Q^{\top}-C_{n}^{1} \operatorname{diag}\left(\lambda_{i}\right)$ must be invertible, and, therefore,

$$
\begin{aligned}
\hat{\boldsymbol{g}}^{(k)}(0)= & -C_{n}^{2}\left[\boldsymbol{Q}^{\top}-C_{n}^{1} \operatorname{diag}\left(\lambda_{i}\right)\right]^{-1} \operatorname{diag}\left(\sigma_{i}^{2}\right) \hat{\boldsymbol{g}}^{(k-2)}(0) \\
& -C_{n}^{1}\left[\boldsymbol{Q}^{\top}-C_{n}^{1} \operatorname{diag}\left(\lambda_{i}\right)\right]^{-1} \operatorname{diag}\left(b_{i}\right) \hat{\boldsymbol{g}}^{(k-1)}(0) .
\end{aligned}
$$

This leads to (3.11). By a similar calculation, we can prove (3.10). 
To end this section, we provide an example, in which the explicit expression of $\hat{\mathbf{g}}(\alpha)$ can be derived. Let

$$
\mathrm{d} X_{t}=-\lambda\left(J_{t}\right) X_{t} \mathrm{~d} t+\sqrt{\lambda\left(J_{t}\right)} \mathrm{d} B_{t}+\mathrm{d} L_{t} ; \quad\left(X_{0}, J_{0}\right)=(x, i), \quad x \geq 0, i \in \mathbb{E},
$$

where $\left\{J_{t}\right\}_{t \geq 0}$ is a continuous-time Markov chain with state space $\mathbb{E}=\{1,2\}$ and intensity matrix

$$
\boldsymbol{Q}=\left(\begin{array}{cc}
-\mu_{1} & \mu_{1} \\
\mu_{2} & -\mu_{2}
\end{array}\right) .
$$

By Theorem 3.2, $\hat{g}_{1}(\alpha)$ and $\hat{g}_{2}(\alpha)$ solve the following equations:

$$
\begin{aligned}
& \left(-\mu_{1}+\frac{\lambda_{1}}{2} \alpha^{2}\right) \hat{g}_{1}(\alpha)+\mu_{2} \hat{g}_{2}(\alpha)-\lambda_{1} \alpha \hat{g}_{1}^{\prime}(\alpha)=p_{1} \alpha, \\
& \mu_{1} \hat{g}_{1}(\alpha)+\left(-\mu_{2}+\frac{\lambda_{2}}{2} \alpha^{2}\right) \hat{g}_{2}(\alpha)-\lambda_{2} \alpha \hat{g}_{2}^{\prime}(\alpha)=p_{2} \alpha .
\end{aligned}
$$

For convenience, let $h(\alpha):=\lambda_{1} \hat{g}_{1}(\alpha)+\lambda_{2} \hat{g}_{2}(\alpha)$ and $p:=p_{1}+p_{2}$. Then (3.12) implies that

$$
\frac{1}{2} \alpha h(\alpha)-h^{\prime}(\alpha)=p .
$$

By the initial condition $h(0)=\left(\lambda_{1} \mu_{2}+\lambda_{2} \mu_{1}\right) /\left(\mu_{1}+\mu_{2}\right), h(\alpha)$ is given by

$$
h(\alpha)=\exp \left\{\frac{1}{4} \alpha^{2}\right\}\left(\frac{\lambda_{1} \mu_{2}+\lambda_{2} \mu_{1}}{\mu_{1}+\mu_{2}}-p \int_{0}^{\alpha} \exp \left\{-\frac{1}{4} s^{2}\right\} \mathrm{d} s\right) .
$$

Note the fact that $h(\alpha) \rightarrow 0$ as $\alpha \rightarrow \infty$. We deduce from (3.13) that

$$
p=\frac{\lambda_{1} \mu_{2}+\lambda_{2} \mu_{1}}{\mu_{1}+\mu_{2}}\left[\int_{0}^{\infty} \exp \left\{-\frac{1}{4} s^{2}\right\} \mathrm{d} s\right]^{-1}=\sqrt{\frac{1}{\pi}} \frac{\lambda_{1} \mu_{2}+\lambda_{2} \mu_{1}}{\mu_{1}+\mu_{2}} .
$$

Substituting (3.13) into (3.12), we obtain

$$
\hat{g}_{1}(\alpha)=\alpha^{-\left(\mu_{1} / \lambda_{1}+\mu_{2} / \lambda_{2}\right)} \exp \left\{\frac{1}{4} \alpha^{2}\right\} \int_{0}^{\alpha} s^{\left(\mu_{1} / \lambda_{1}+\mu_{2} / \lambda_{2}\right)} \exp \left\{-\frac{1}{4} s^{2}\right\}\left[\frac{\mu_{2}}{\lambda_{1} \lambda_{2} s} h(s)-\frac{p_{1}}{\lambda_{1}}\right] \mathrm{d} s
$$

and

$\hat{g}_{2}(\alpha)=\alpha^{-\left(\mu_{1} / \lambda_{1}+\mu_{2} / \lambda_{2}\right)} \exp \left\{\frac{1}{4} \alpha^{2}\right\} \int_{0}^{\alpha} s^{-\left(\mu_{1} / \lambda_{1}+\mu_{2} / \lambda_{2}\right)} \exp \left\{-\frac{1}{4} s^{2}\right\}\left[\frac{\mu_{1}}{\lambda_{1} \lambda_{2} s} h(s)-\frac{p_{2}}{\lambda_{2}}\right] \mathrm{d} s$.

It is easy to see that $\hat{g}_{1}(\alpha) \rightarrow \mu_{2} /\left(\mu_{1}+\mu_{2}\right)$ and $\hat{g}_{2}(\alpha) \rightarrow \mu_{1} /\left(\mu_{1}+\mu_{2}\right)$ as $\alpha \rightarrow 0$. Finally, we are left to find $p_{1}$ and $p_{2}$. We note that $\hat{g}_{1}(\alpha), \hat{g}_{2}(\alpha) \rightarrow 0$ as $\alpha \rightarrow \infty$, which suggests that

$$
\int_{0}^{\infty} s^{-\left(\mu_{1} / \lambda_{1}+\mu_{2} / \lambda_{2}\right)} \exp \left\{-\frac{1}{4} s^{2}\right\}\left[\frac{\mu_{2}}{\lambda_{1} \lambda_{2} s} h(s)-\frac{p_{1}}{\lambda_{1}}\right] \mathrm{d} s=0
$$

and

$$
\int_{0}^{\infty} s^{-\left(\mu_{1} / \lambda_{1}+\mu_{2} / \lambda_{2}\right)} \exp \left\{-\frac{1}{4} s^{2}\right\}\left[\frac{\mu_{1}}{\lambda_{1} \lambda_{2} s} h(s)-\frac{p_{2}}{\lambda_{2}}\right] \mathrm{d} s=0 .
$$


Then a straightforward calculation gives

$p_{1}=\frac{\mu_{2}}{\lambda_{2}}\left[\int_{0}^{\infty} s^{-\left(\mu_{1} / \lambda_{1}+\mu_{2} / \lambda_{2}\right)} \exp \left\{-\frac{1}{4} s^{2}\right\} \mathrm{d} s\right]^{-1} \int_{0}^{\infty} s^{\left(\mu_{1} / \lambda_{1}+\mu_{2} / \lambda_{2}-1\right)} \exp \left\{-\frac{1}{4} s^{2}\right\} h(s) \mathrm{d} s$

and

$p_{2}=\frac{\mu_{1}}{\lambda_{1}}\left[\int_{0}^{\infty} s^{-\left(\mu_{1} / \lambda_{1}+\mu_{2} / \lambda_{2}\right)} \exp \left\{-\frac{1}{4} s^{2}\right\} \mathrm{d} s\right]^{-1} \int_{0}^{\infty} s^{\left(\mu_{1} / \lambda_{1}+\mu_{2} / \lambda_{2}-1\right)} \exp \left\{-\frac{1}{4} s^{2}\right\} h(s) \mathrm{d} s$.

Thus, together with the values of $h(s), p_{1}$, and $p_{2},(3.14)$ and (3.15) jointly give the values of $\hat{g}_{1}(\alpha)$ and $\hat{g}_{2}(\alpha)$.

\section{References}

[1] Applebaum, D. (2004). Lévy Processes and Stochastic Calculus. Cambridge University Press.

[2] Bertoin, J. (1996). Lévy Processes. Cambridge University Press.

[3] De Saporta, B. and Yao, J.-F. (2005). Tail of a linear diffusion with Markov switching. Ann. Appl. Prob. 15, 992-1018.

[4] Golub, G. H. and Van Loan, C. F. (1983). Matrix Computations. Johns Hopkins University Press, Baltimore, MD.

[5] GuYon, X., IovlefF, S. AND Yao, J.-F. (2004). Linear diffusion with stationary switching regime. ESAIM Prob. Statist. 8, 25-35.

[6] Harrison, J. M. (1985). Brownian Motion and Stochastic Flow Systems. John Wiley, New York.

[7] Novikov, A. A. (2004). Martingales and first-exit times for the Ornstein-Uhlenbeck process with jumps. Theory Prob. Appl. 48, 288-303.

[8] Oberhettinger, F. And Badi, L. (1973). Tables of Laplace Transforms. Springer, New York.

[9] PAtie, P. (2005). On a martingale associated to generalized Ornstein-Uhlenbeck processes and an application to finance. Stoch. Process. Appl. 115, 593-607.

[10] SRIKANt, R. AND Whitt, W. (1996). Simulation run lengths to estimate blocking probabilities. ACM Trans. Model. Comput. Simul. 6, 7-52.

[11] Ward, A. R. and Glynn, P. W. (2003). A diffusion approximation for a Markovian queue with reneging. Queueing Systems 43, 103-128.

[12] Ward, A. R. And Glynn, P. W. (2003). Properties of the reflected Ornstein-Uhlenbeck process. Queueing Systems 44, 109-123.

[13] Ward, A. R. And Glynn, P. W. (2005). A diffusion approximation for a GI/GI/1 queue with balking or reneging. Queueing Systems 50, 371-400. 